\title{
Особенности задающего источника ModBox Front-End для генерации наносекундных импульсов
}

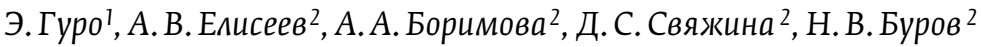 \\ 1 iXblue Photonics, Ланьон Франция \\ 2 АО «ЛЛС», Санкт-Петербург, Россия
}

\begin{abstract}
Система ModBox Front-End предназначена для использования в качестве задающего источника в лазерных установках, генерирующих излучение с высокой плотностью энергии. В обзоре представлены новые программные функции устройства и его новейшие усовершенствования: обновленная аппаратная часть оптического и РЧ усилителей, увеличенная выходная мощность, стабильность, улучшенные характеристики аппаратного формирования импульса.
\end{abstract}

Ключевые слова: формирование оптических импульсов, ModBox-FrontEnd, iXblue

\section{Features of the ModBox Front-End Master Source for Generating Nanosecond Pulses}

\author{
H. Gouraud', A. V. Eliseev², A. A. Borimova², D.S.Svyazina², N. V. Burov² \\ 1 iXblue Photonics, Lannion, France \\ 2 JSC “LLS”, St. Petersburg, Russia
}

\begin{abstract}
The ModBox Front-End system is intended for use as a master source in laser systems generating radiation with a high energy density. The review presents new software features of the device and its latest enhancements: updated hardware of optical and RF amplifiers, increased output power, stability, improved characteristics of hardware pulse shaping.
\end{abstract}

Keywords: Pulse shaping, ModBox-FrontEnd, iXblue

B ысокомощные лазерные системы применяются во многих областях технологий и науки, таких как лазерная имплозия, термоядерный синтез, взаимодействие лазерного излучения с веществом и много другое [1, 2]. В подобных практических приложениях важной задачей является контроль параметров лазерного излучения. Обладая обширными познаниями в области проектирования систем модуляции, компания iXblue Photonics предлагает техническое решение - систему ModBox Front-End, которое демонстрирует высокие результаты и обеспечивает удобство в использовании.
ModBox Front-End - задающий источник для лазерных установок высокой мощности, который обеспечивает генерацию оптических импульсов с высоким коэффициентом экстинкции. Устройство (рис. 1) позволяет регулировать форму, длительность импульса (125 пс - 100 нс) и частоту их повторения. Генерация коротких импульсов осуществляется за счет высокопроизводительного непрерывного лазерного источника в сочетании с каскадом модуляции с широкой полосой пропускания на основе электрооптического модулятора $\mathrm{LiNbO}_{3}$ (ниобат лития) [3]. 


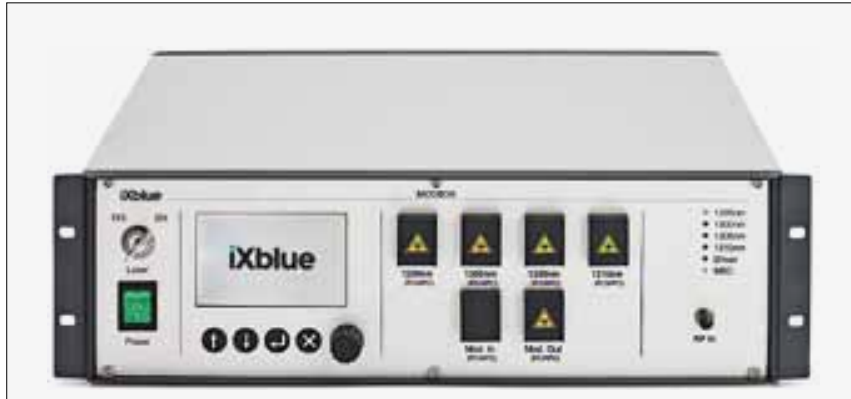

Puc. 1. Cucmema ModBox Front-End [4]

\section{ФУНКЦИЯ ФОРМИРОВАНИЯ ИМПУЛЬСОВ ОПТИЧЕСКОГО ИЗЛУЧЕНИЯ}

Возможность формировать импульс во времени имеет очень важное значение при создании импульсного лазерного источника. Поэтому появление на рынке устройств для формирования наносекундных импульсов системы ModBox Front-End стало настоящим вызовом существующим технологиям.

В РЧ усилитель данной модели встроен генератор электрических сигналов произвольной формы (AWG) (рис. 2). Его конструкция построена на монолитных интегральных микроволновых микросхемах последнего поколения, которые обеспечивают высокую линейность характеристик. Кроме этого РЧ-усилитель имеет широкий рабочий диапазон, что позволяет сократить время нарастания и спада импульса. Это дает возможность усиливать импульсы в диапазоне длительностей от субнаносекунд до микросекунд.

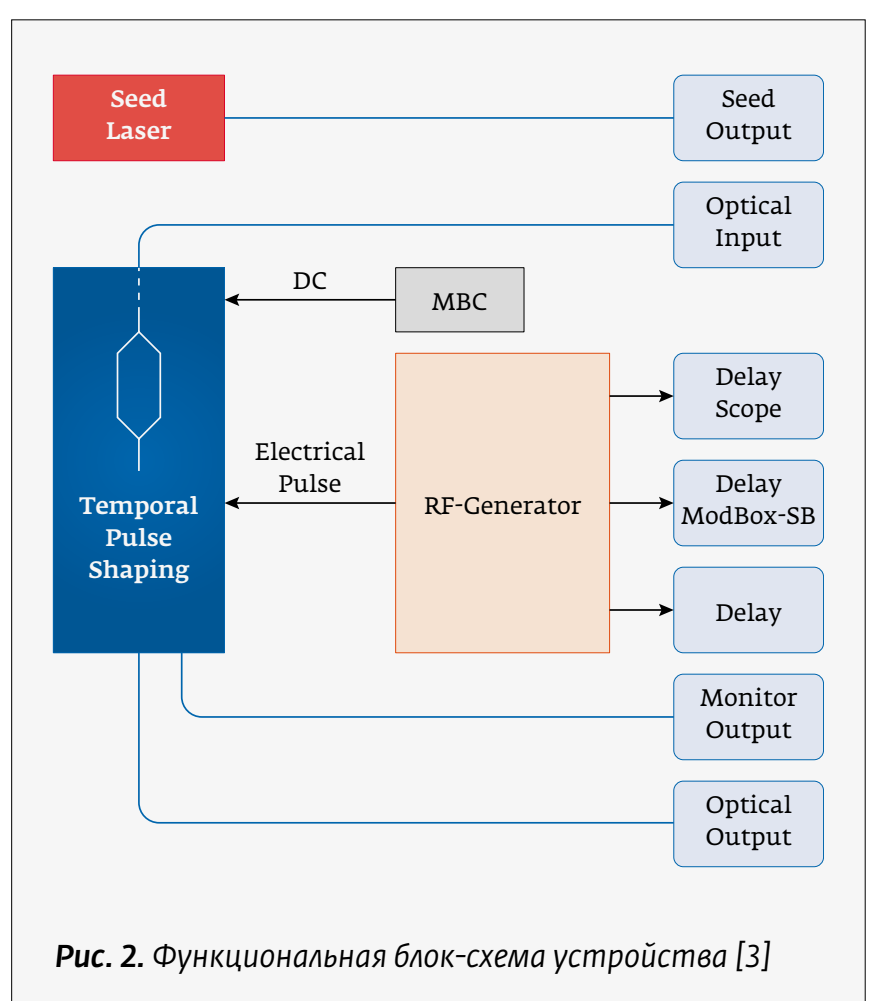

Система учитывает передаточную функцию электрооптического модулятора (ЭОМ) (синусоидальный отклик): в программном обеспечении ModBox регистрируется обратный отклик ЭОМ. Таким образом, пользователь задает желаемую форму оптического импульса, а система генерирует в реальном времени точную и ожидаемую форму импульса. На рис. 3 представлены кадры
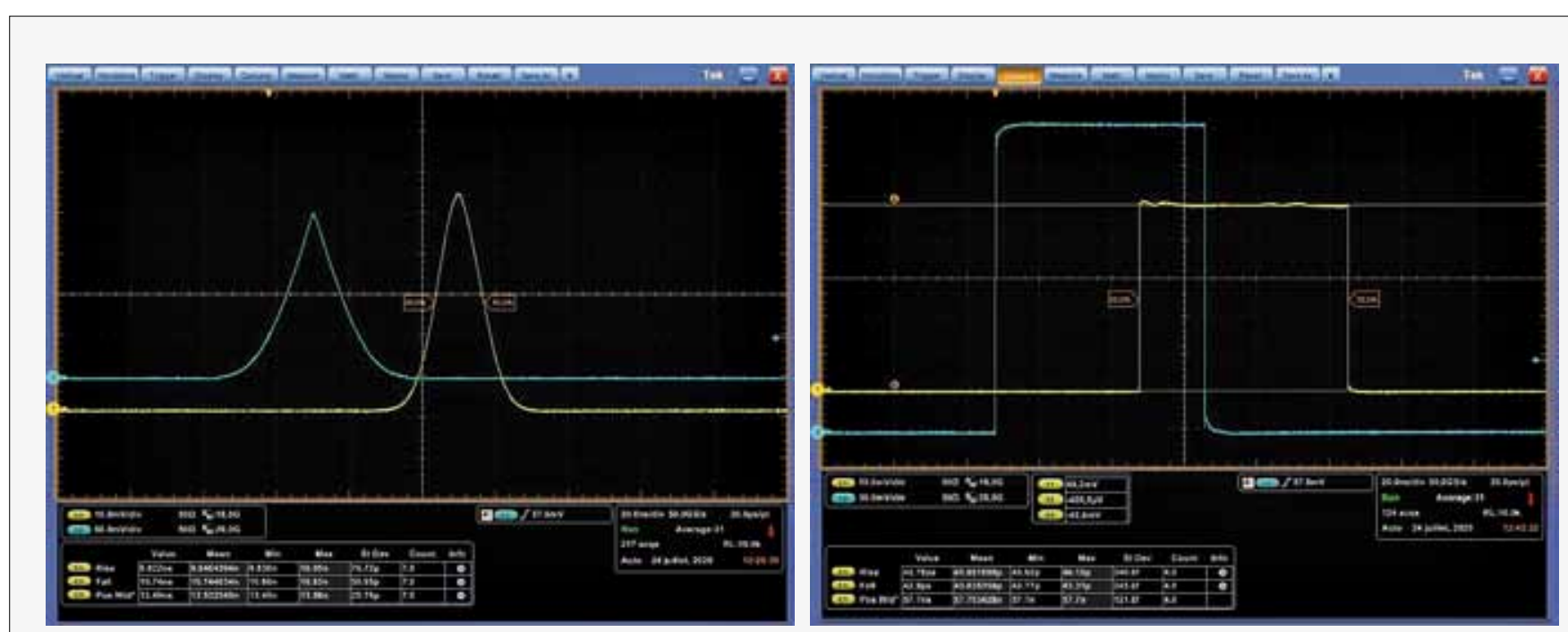

Puс. 3. Кадры экрана интерфейса: желтые кривые - формы импульсов, заданные пользователем; синие иинии - формы сигналов, создаваемые генератором электрических сигналов (AWG) [4] 

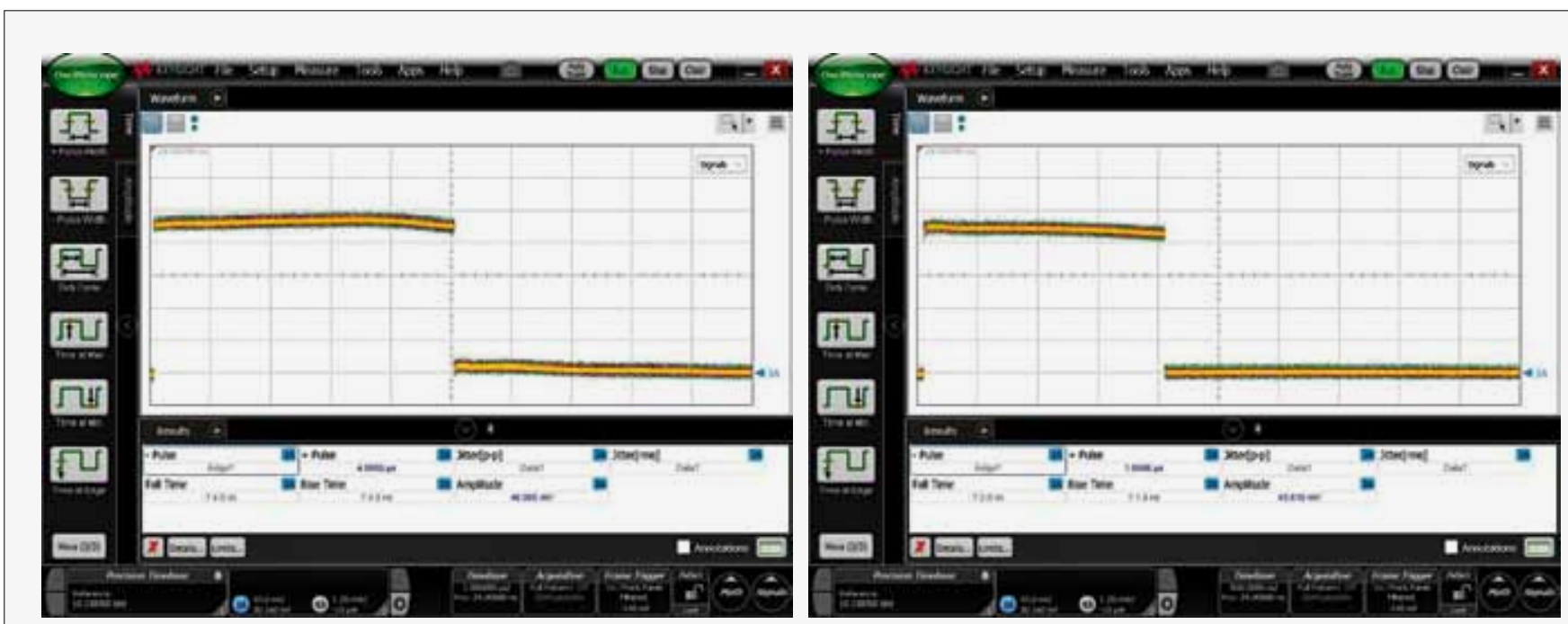

Pис. 4. Отклик на оптические импульсы длительностью в несколько микросекунд [4]

экрана интерфейса, где желтые кривые - это оптические импульсы, заданные пользователем, а синие линии соответствуют формам сигналов, генерируемых AWG.

ModBox использует интерфейс последнего поколения, это обеспечивает быструю загрузку, изменение и обновление формы импульса. Подчеркнем тот факт, что форму сигнала можно изменять в режиме реального времени без прерывания работы системы (рис. 4). Более того, изменение формы волны не вызывает пропадания оптических импульсов или значительных колебаний выходной энергии, кроме изменения энергии, вызванного самой регулировкой. Такая возможность обеспечена тем фактом, что на уровень выходной оптической мощности влияет работа только ЭОМ (который связан с AWG и изменением формы сигнала).

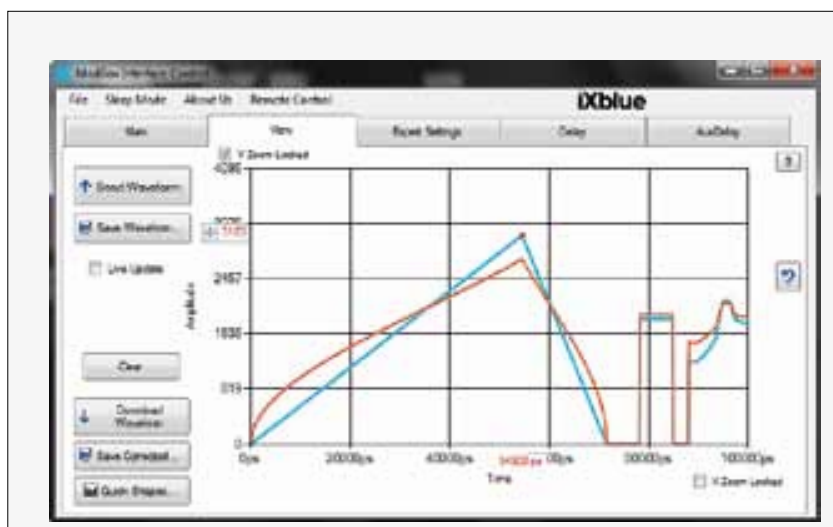

Puc. 5. Коррекция формы импульса [4]

\section{МАКСИМАЛЬНАЯ ПИКОВАЯ МОЩНОСТЬ СИСТЕМЫ MODBOX}

Работа системы ModBox Front-End базируется на сочетании мощногго усилителя и ЭОМ высокой оптической мощности. Используемый модулятор был выбран из-за низких потерь, вносимых им в работу всей системы. При сборке системы особое внимание уделяется качеству сращивания волоконных компонентов. Поэтому можно ожидать, что узлы системы будут вносить минимальные потери в бюджет оптической мощности.

Поскольку усилитель и оптический модулятор функционируют в своих стандартных рабочих условиях, характеристики системы (стабильность мощности и энергии), а также ее надежность не ухудшаются. Действительно, ModBox Front-End преодолевает минимальное значение пиковой мощности, равное 600 мВт (эквивалент 6 нДж при 10 нс), и типичное значение 860 мВт (эквивалентно 8,6 нДж при 10 нс) достигается без отрицательного влияния на другие параметры (рис. 5).

На рис. 6 представлена зависимость величины средней мощность от времени. Как видно из графика, постоянство значения величины $(P=0,860$ мВт) отражает стабильность работы системы. Работа в "комфортной зоне" вне предела порога повреждения обеспечивает гарантийный срок службы системы.

\section{ЗАКЛЮЧЕНИЕ}

Система ModBox Front-End представляет готовое решение для конкретного класса лазерных устройств: лазеров с высокой энергией или с высо- 


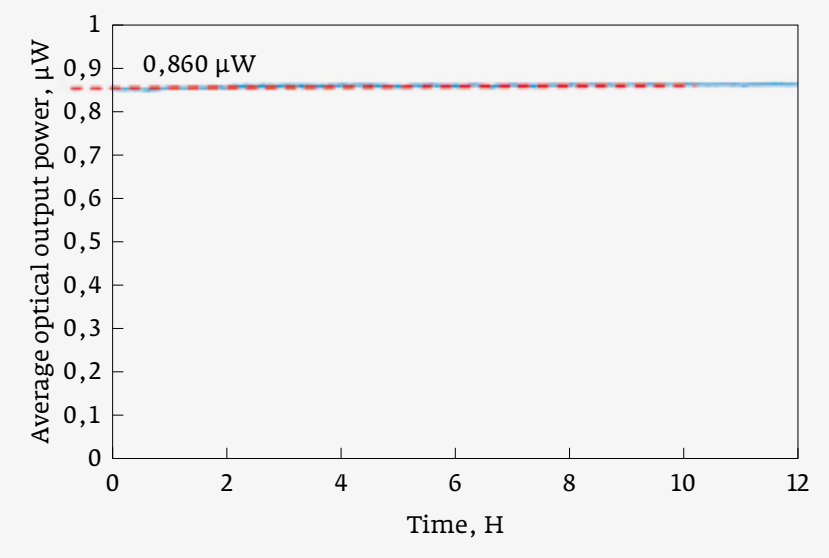

Puc. 6. Средняя выходная мощность дяя импульсов длительностью 10 нс при частоте 100 Гц [4]

кой мощностью излучения. Система используется в цепях мощных усилителей, чтобы создать задающий лазер или ОРСРА лазер накачки. Применения системы простираются от научных исследований и образовательной деятельности до промышленных заказчиков. ModBox Front-End также находит применение в таких областях, как ускоре-
AО "ЛЛС" является дистрибьютором компании iXblue Photonics на территории Российской Федерации и стран Таможенного Союза и предлагает наиболее выгодные условия поставки продукции, полную техническую поддержку, а также поставку образцов. Получить дополнительную информацию вы можете на сайте производителя ixblue Photonics или обратившись в компанию "ллс".

ние частиц, инерциально-термоядерный синтез, лучевая терапия, генерация рентгеновских лучей, тестирование датчиков, лазерное ударное сжатие для лазерной шлифовки или динамического сжатия.

\section{REFERENCES}

1. Hecht J. High Power Laser Applications. Understanding Lasers: An Entry Level Guide, Fourth Edition. 2018.

2. Svanberg S. High-Power Lasers and their Applications. Advances in Quantum Chemistry. 1998; 30: 209-233.

3. H. Gouraud. ModBox-Front-End - The Latest Improvement, 2020 / URL: https:// www.industrialtechnology.co.uk/products.html?comp[L]=L\&comp[O]=Q.

4. URL: www.photonics.ixblue.com.

\section{ЭССЕНТОПТИКС (|() ESSENTOPTICS}

\section{PHOTON RT}

\section{Спектрофотометр для Оптиков I Spectrophotometer for Coaters}

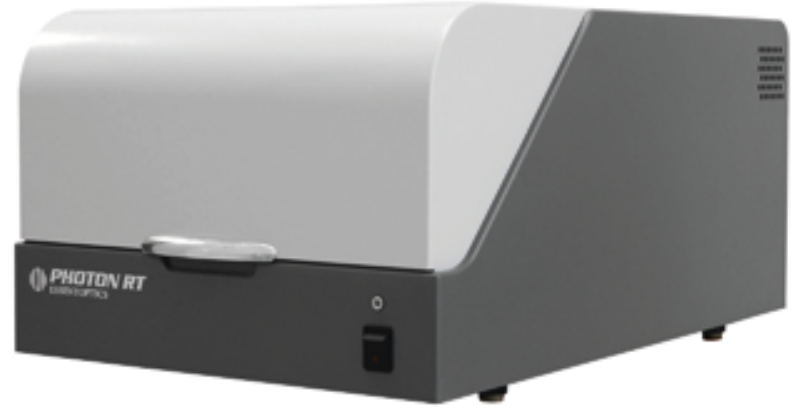

$$
185-5200 \mathrm{~nm}
$$

\section{УНИКАЛЬНО ШИРОКИЙ СПЕКТРАЛЬНЫЙ ДИАПАЗОН В ОДНОМ ПРИБОРЕ}

WORLD-RECORD WAVELENGTH RANGE IN ONE INSTRUMENT 